\title{
MULTI-PHASE NATURE OF A RADIATION-DRIVEN FOUNTAIN WITH NUCLEAR STARBURST IN A LOW-MASS ACTIVE GALACTIC NUCLEUS
}

\author{
Keilchi Wada ${ }^{1,4}$, Marc Schartmann ${ }^{2}$, and Rowin Meijerink ${ }^{3}$ \\ ${ }^{1}$ Kagoshima University, Kagoshima 890-0065, Japan; wada@astrophysics.jp \\ ${ }^{2}$ Centre for Astrophysics and Supercomputing, Swinburne University of Technology, P.O. Box 218, Hawthorn, VIC 3122, Australia \\ ${ }^{3}$ Leiden Observatory, P.O. Box 9513, NL-2300 RA Leiden, The Netherlands \\ Received 2016 July 20; revised 2016 August 19; accepted 2016 August 24; published 2016 September 9
}

\begin{abstract}
The structures and dynamics of molecular, atomic, and ionized gases are studied around a low-luminosity active galactic nucleus (AGN) with a small $\left(2 \times 10^{6} M_{\odot}\right)$ black hole using three-dimensional (3D) radiationhydrodynamic simulations. We studied, for the first time, the non-equilibrium chemistry for the X-raydominated region in the "radiation-driven fountain" with supernova feedback. A double hollow cone structure is naturally formed without postulating a thick "torus" around a central source. The cone is occupied with an inhomogeneous, diffuse ionized gas and surrounded by a geometrically thick $(h / r \gtrsim 1)$ atomic gas. Dense molecular gases are distributed near the equatorial plane, and energy feedback from supernovae enhances their scale height. Molecular hydrogen exists in a hot phase $(>1000 \mathrm{~K})$ as well as in a cold $(<100 \mathrm{~K})$, dense $\left(>10^{3} \mathrm{~cm}^{-3}\right.$ ) phase. The velocity dispersion of $\mathrm{H}_{2}$ in the vertical direction is comparable to the rotational velocity, which is consistent with near-infrared observations of nearby Seyfert galaxies. Using 3D radiation transfer calculations for the dust emission, we find polar emission in the mid-infrared band (12 $\mu \mathrm{m})$, which is associated with bipolar outflows, as suggested in recent interferometric observations of nearby AGNs. If the viewing angle for the nucleus is larger than $75^{\circ}$, the spectral energy distribution is consistent with that of the Circinus galaxy. The multi-phase interstellar medium observed in optical/infrared and X-ray observations is also discussed.
\end{abstract}

Key words: galaxies: active - galaxies: ISM - galaxies: nuclei

\section{INTRODUCTION}

Recent observations of nearby active galactic nuclei (AGNs) suggest that the long-standing standard picture, i.e., bright emission from a central source is obscured by a dusty "donutlike" torus, needs to be reconsidered. For example, midinfrared (MIR) interferometric observations showed that the bulk of MIR is emitted from the dust in the polar region, not from the dusty torus (Tristram et al. 2014; Asmus et al. 2016). However, it is not clear how this MIR emission is related to the double (or single) hollow cones of ionized gas that are often observed in many AGNs. The observed properties of AGNs are related to the conditions of the interstellar medium (ISM) near the nucleus, but its multi-phase structure in the context of the standard picture is still unclear.

Recently, we have proposed a novel mechanism of the obscuring structures around AGNs in which outflowing and inflowing gases are driven by radiation from the accretion disk, forming a geometrically thick disk on the scale of a few to tens of parsecs (Wada 2012, hereafter W12). The quasi-steady circulation of gas, i.e., the "radiation-driven fountain," may obscure the central source, and thereby the differences in the spectral energy distributions (SEDs) of typical type-1 and type2 Seyfert galaxies are consistently explained (Schartmann et al. 2014) (hereafter Sch14). We also showed in Wada (2015, hereafter W15) that the observed properties of obscured AGNs change as a function of their luminosity due to the fountain flows, which is consistent with recent observations (e.g., Burlon et al. 2011; Lusso et al. 2013; Ueda et al. 2014; Aird et al. 2015; Buchner et al. 2015).

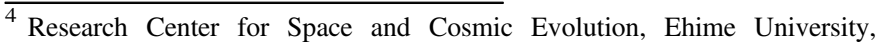
Matsuyama 790-8577, Japan.
}

There are many instances in which AGNs are associated with circumnuclear starbursts (e.g., Davies et al. 2007; Chen et al. 2009; Hicks et al. 2009; Imanishi et al. 2011; Diamond-Stanic \& Rieke 2012; Woo et al. 2012; Durré \& Mould 2014; Esquej et al. 2014; Bernhard et al. 2016). The nuclear starbursts themselves could inflate the circumnuclear disk and obscure the central source (Wada \& Norman 2002; Wada et al. 2009). Therefore, the interplay between the radiation-driven fountain and the feedback from nuclear starbursts should be an important process to be understood for the multi-phase nature of the ISM around AGNs.

In this Letter, we apply the radiation-driven fountain model to the case of a low black hole $(\mathrm{BH})$ mass $\left(\sim 10^{6} M_{\odot}\right)$, which was not studied in our previous papers. We expect that the nuclear starburst would have more impact on structures of the circumnuclear disk around smaller BHs (Wada \& Norman 2002; Wada 2004). For the first time, we solve the X-ray-dominated region (XDR) chemistry with three-dimensional (3D) gas dynamics under the effect of the radiation from AGNs and and the energy feedback from supernovae ( $\mathrm{SNe}$ ). The selected parameters are close to those in the nearest Seyfert galaxy, the Circinus galaxy, and we compare the results with multi-wavelength observations using $3 \mathrm{D}$ radiative transfer calculations.

\section{NUMERICAL METHODS AND MODELS}

\subsection{Numerical Methods}

We follow the same numerical methods as those given in W12 and W15, i.e., 3D Eulerian hydrodynamic code with a uniform grid that accounts for radiative feedback processes from the central source using a ray-tracing method. One major difference from our previous papers is that we include the nonequilibrium XDR chemistry (Maloney et al. 1996; Meijerink \& 
Spaans 2005) for $256^{3}$ zones (resolution of $0.125 \mathrm{pc}$ ). In the present models, self-gravity of the gas is ignored because it is not essential for the gas dynamics in the radiation-driven fountain (see also Namekata \& Umemura 2016). The supernova feedback is implemented based on Wada (2001), in which the energy from a supernova is injected in a randomly selected grid cell near the equatorial plane.

We assumed that the radiation flux caused by the accretion disk is non-spherical (see below), and the flux is calculated for all grid cells using $256^{3}$ rays. The outflows are driven mainly by the radiation pressure of dust and X-ray heating. A cooling function for $20 \mathrm{~K}<T_{\mathrm{gas}}<10^{8} \mathrm{~K}$ (Meijerink \& Spaans 2005; Wada et al. 2009) and solar metallicity are assumed. We include photoelectric heating due to a uniform far-ultraviolet (FUV) field with $G_{0}=1000$, where $G_{0}$ is the incident FUV field normalized to the local interstellar value.

If temperature of the dust irradiated by a central source $T_{\text {dust }}$ exceeds $1500 \mathrm{~K}$, we assume that the dust is sublimated, and no dust is assumed for $T_{\mathrm{gas}}>10^{5} \mathrm{~K}$ due to dust sputtering. Here, we assume that the ISM in the central several tens of parsecs is optically thin with respect to the re-emission from the hot dust. The SED of the AGN and the dust absorption cross-sections are taken from Laor \& Draine (1993). In the hydrodynamic calculations, the dust is not treated as an independent component, but assumed to move together with the gas. We use a standard galactic dust model (Schartmann et al. 2011) with a dust-to-gas ratio of 0.01 .

We used a selection of reactions from the chemical network described by Meijerink \& Spaans (2005) and Ádámkovics et al. (2011) for 25 species: $\mathrm{H}, \mathrm{H}_{2}, \mathrm{H}^{+}, \mathrm{H}_{2}^{+}, \mathrm{H}_{3}^{+}, \mathrm{H}^{-}, \mathrm{e}^{-}, \mathrm{O}, \mathrm{O}_{2}, \mathrm{O}^{+}$, $\mathrm{O}_{2}^{+}, \mathrm{O}_{2} \mathrm{H}^{+}, \mathrm{OH}, \mathrm{OH}^{+}, \mathrm{H}_{2} \mathrm{O}, \mathrm{H}_{2} \mathrm{O}^{+}, \mathrm{H}_{3} \mathrm{O}^{+}, \mathrm{C}, \mathrm{C}^{+}, \mathrm{CO}, \mathrm{Na}$, $\mathrm{Na}^{+}, \mathrm{He}, \mathrm{He}^{+}$, and $\mathrm{HCO}^{+}$. At every time step, the gas density, gas and dust temperatures, and ionization parameters in the $256^{3}$ grid cells are passed to the chemistry module. The chemistry module returns the abundances of the species, and they are advected based on the gas velocity. ${ }^{5}$

\subsection{Initial Conditions and Model Setup}

In contrast to our previous papers (W12, W15, and Sch14), we assume a smaller black hole mass $\left(2 \times 10^{6} M_{\odot}\right)$ in this study, and other parameters are selected to fit with the nearest AGN, the Circinus galaxy. Then the numerical results can be directly compared with observations (see Section 4). We follow the evolution of a rotating gas disk (total gas mass is $2 \times 10^{6} M_{\odot}$ ) in a fixed spherical gravitational potential under the influence of radiation from the central source (the Eddington ratio of 0.2 and the bolometric luminosity of $L_{\mathrm{bol}}=5 \times 10^{43} \mathrm{erg} \mathrm{s}^{-1}$ are fixed during the calculation). We assume a time-independent external potential $\Phi_{\mathrm{ext}}(r) \equiv$ $-(27 / 4)^{1 / 2}\left[v_{1}^{2} /\left(r^{2}+a_{1}^{2}\right)^{1 / 2}+v_{2}^{2} /\left(r^{2}+a_{2}^{2}\right)^{1 / 2}\right]$, where $a_{1}=$ $100 \mathrm{pc}, a_{2}=2.5 \mathrm{kpc}, v_{1}=147 \mathrm{~km} \mathrm{~s}^{-1}$, and $v_{2}=147 \mathrm{~km} \mathrm{~s}^{-1}$. To prepare quasi-steady initial conditions without radiative feedback, we first evolve axisymmetric and rotationally supported thin disks with uniform density profiles. After the thin disks are dynamically settled, the radiation feedback and $\mathrm{SNe}$ feedback (the supernova rate is a free parameter and is fixed during the calculation) are turned on.

The ultraviolet flux is assumed to be $F_{\mathrm{UV}}(\theta) \propto$ $\cos \theta(1+2 \cos \theta)$ (Netzer 1987), where $\theta$ denotes the angle

\footnotetext{
To save computational memory, we follow the advection only for $\mathrm{H}, \mathrm{H}^{+}$, $\mathrm{H}_{2}, \mathrm{O}, \mathrm{O}^{+}, \mathrm{H}_{2} \mathrm{O}, \mathrm{OH}, \mathrm{C}, \mathrm{C}^{+}$, and $e^{-}$.
}

from the rotational axis (z-axis). The $\mathrm{X}$-ray radiation, on the other hand, is assumed to be spherically symmetric (Netzer 1987; Xu 2015). The UV and X-ray fluxes are calculated from the bolometric luminosity (Marconi et al. 2004). The total $\mathrm{X}$-ray luminosity $(2-10 \mathrm{keV})$ is $L_{X}=2.8 \times 10^{42} \mathrm{erg} \mathrm{s}^{-1}$.

Although the calculations are fully $3 \mathrm{D}$ (i.e., without assuming any symmetries), the direction of the emerging non-spherical radiation is assumed to be parallel to the rotational axis of the circumnuclear gas disk in the present model (see W15 for non-parallel cases).

\section{RESULTS}

Figure 1 shows the distributions of three phases, i.e., atomic, molecular, and ionized hydrogen, as well as the gas temperature in a quasi-steady state. The distribution of neutral hydrogen $\left(\mathrm{H}^{0}\right)$ shows a hollow cone structure surrounded by a geometrically thick disk, similar to that typically observed in the radiation-driven fountain models (W12; W15). The bipolar outflow of the low-density gas is directly caused by the radiation pressure and X-ray heating due to the AGN radiation.

The high-density molecular gas forms a clumpy disk similar to that in the previous starburst model (Wada \& Norman 2002; Wada et al. 2009). A clumpy disk of $\mathrm{H}_{2}$ is embedded in the geometrically thick disk of $\mathrm{H}^{0}$. Here, $\mathrm{H}^{+}$is mostly distributed outside the thick neutral disk and in the bipolar outflows, which are deficient in $\mathrm{H}^{0}$ and $\mathrm{H}_{2}$. The maximum velocity of the outflow is $\sim 500 \mathrm{~km} \mathrm{~s}^{-1}$ near the center, but it slows down to $\sim 100 \mathrm{~km} \mathrm{~s}^{-1}$ in the outer region. In the majority of the highdensity thin disk, the temperature of the gas is less than $100 \mathrm{~K}$, except for the hot regions caused by SNe.

Figure 2 shows the mass-weighted histogram of $\mathrm{H}_{2}$ and $\mathrm{H}^{+}$ as a function of their number density and temperature. We find that $\mathrm{H}_{2}$ mostly exists in the low-temperature $\left(T_{\mathrm{gas}}<100 \mathrm{~K}\right)$ and high-density $\left(n>100 \mathrm{~cm}^{-1}\right)$ phase. On the other hand, $\mathrm{H}^{+}$ is dominant in the hot gas around $T_{\mathrm{gas}} \sim 8000 \mathrm{~K}$. There are two additional phases in the $\mathrm{H}^{+}$gas around $\mathrm{T}_{\text {gas }} \sim 30,000 \mathrm{~K}$ and $5000-7000 \mathrm{~K}$ with $n \sim 10^{2}-10^{3} \mathrm{~cm}^{-1}$. These phases correspond to high-density gases heated by the central radiation near the nucleus $(r \lesssim 5 \mathrm{pc})$ and the disk gas heated by the $\mathrm{SNe}$ feedback, respectively. In Figure 2(a), hot $\mathrm{H}_{2}\left(T_{\text {gas }} \sim 1000\right.$ $3000 \mathrm{~K}$ ) is observed around $n \sim 10-10^{3} \mathrm{~cm}^{-3}$. The total $\mathrm{H}_{2}$ mass in this phase is $4.7 \times 10^{2} M_{\odot}$.

The ratio between the rotational velocity $\left(v_{c}\right)$ and the vertical velocity dispersion $\left(\sigma_{z}\right)$ of hot $(>1000 \mathrm{~K}) \mathrm{H}_{2}$ is plotted as a function of the radius in Figure 3. The ratio declines toward $v_{c} / \sigma_{z} \sim 1$ at larger radii, reflecting the large scale height of the disk (Figure 1) is supported by energy input due to $\mathrm{SNe}$. On the other hand, the ratio remains large $(\gtrsim 10)$ for the pure radiationfountain model without $\mathrm{SNe}$ (dashed line). In the inner region $(r<2 \mathrm{pc})$, the vertical velocity dispersion is negligibly small compared to $v_{c}$ for the model without SN feedback, suggesting the radiation-driven fountain alone does not work to enhance the disk thickness for this low-luminosity AGN (see also W15). The energy feedback from $\mathrm{SNe}$ allows us to keep the hot $\mathrm{H}_{2}$ disk at a thickness of $\sim r / 10$, even at $r<8 \mathrm{pc}$.

Using Very Large Telescope (VLT)/SINFONI and Keck/ OSIRIS, Hicks et al. (2009) found that most local AGNs are associated with a geometrically thick, hot $\mathrm{H}_{2}$ disk in the central several tens of parsecs. They found that the molecular gas is spatially mixed with the nuclear stellar population, and $v_{c} / \sigma_{z} \simeq 1$ for the molecular gas, suggesting a geometrically thick disk that could obscure the nucleus. These observations 


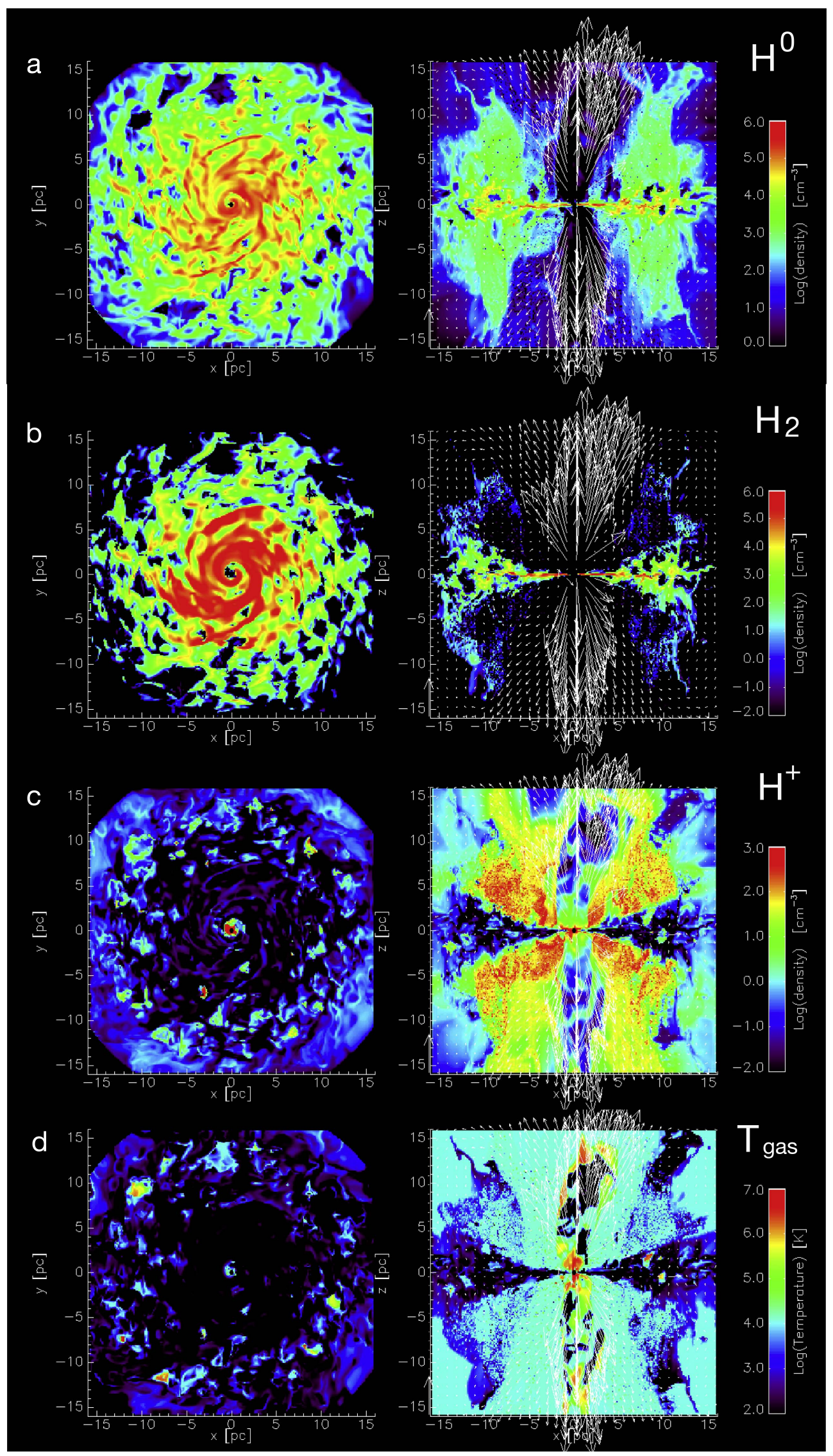

Figure 1. Distributions of (a) $\mathrm{H}^{0}$, (b) $\mathrm{H}_{2}$, (c) $\mathrm{H}^{+}$, and (d) gas temperature on the equatorial plane and $x-z$ plane. The arrows represent velocity vectors of the gas. The average supernova rate is $0.014 \mathrm{yr}^{-1}$. 


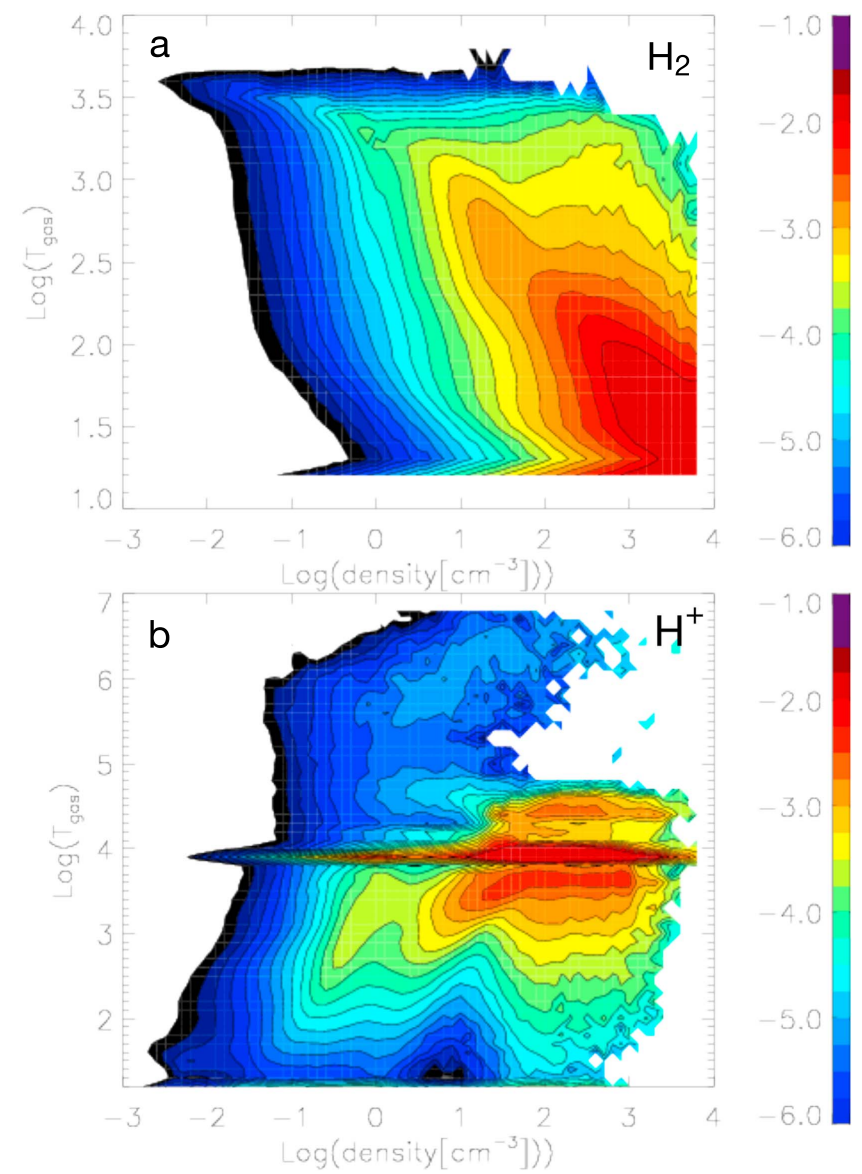

Figure 2. (a) Phase-diagram for molecular hydrogen and (b) for ionized hydrogen. The contours represent mass-weighted abundances of $\mathrm{H}_{2}$ or $\mathrm{H}^{+}$ relative to their maximum values.

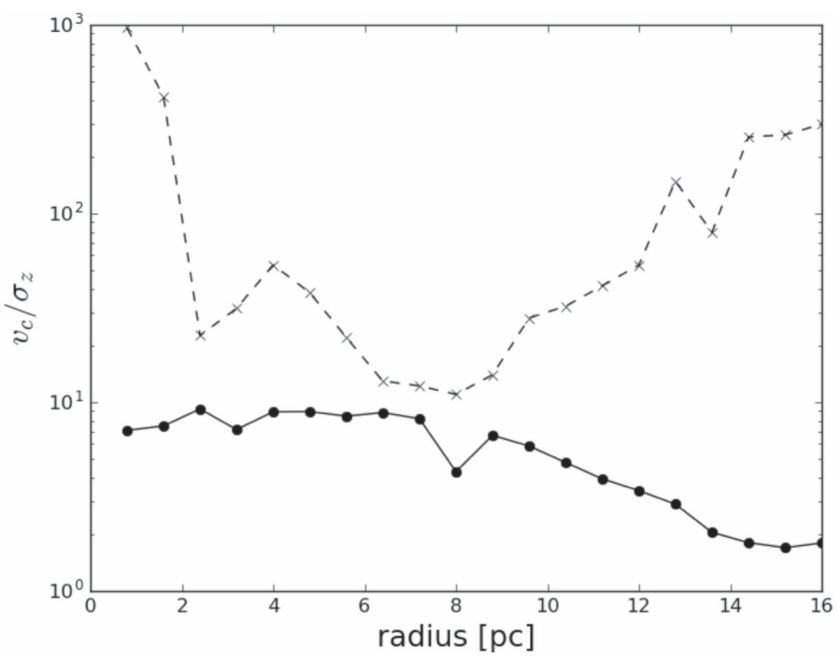

Figure 3. Radial distribution of $v_{c} / \sigma_{z}$, where $\sigma_{z}$ is the velocity dispersion for the $z$-direction and $v_{c}$ is the rotational velocity weighted to the hot $(>1000 \mathrm{~K})$ molecular hydrogen density: with SNe feedback (thick line) and without feedback (dashed line).

are consistent with the structure in the outer part of our model with the SN feedback.

We found that $\mathrm{CO}$ and $\mathrm{C}^{0}$ are distributed similarly to $\mathrm{H}_{2}$ and $\mathrm{H}^{0}$, respectively, as shown in Figure 1. On the other hand, $\mathrm{H}_{2} \mathrm{O}$ mostly concentrates in a thin disk at $r \lesssim 5$ pc (Figure 4(a)).
This is also the case for the $\mathrm{OH}$ molecule. The column density distributions for various species are plotted as a function of the viewing angle in Figure 4(b). We found that the opening angles of the ionized and atomic gas disk are small for a given column density; in other words, their scale heights are large. On the other hand, molecular gases are more concentrated in the thin disk. If the supernova feedback is not included, then the molecular gases form a thin, dense disk (dashed lines).

\section{COMPARISON WITH THE NEAREST SEYFERT GALAXY}

The recent development of new observational methods enables us to illuminate the internal structures of nearby AGNs, especially by using infrared and submillimeter/millimeter interferometers such as VLTI, Keck, and the Atacama Large Millimeter/submillimeter Array (ALMA). The nearest wellobserved prototypical Seyfert 2 galaxy is the Circinus galaxy. Its black hole mass suggested by $\mathrm{H}_{2} \mathrm{O}$ kinematics is $1.7 \pm 0.3 \times 10^{6} M_{\odot}$ (Greenhill et al. 2003), which is a few orders of magnitude smaller than those in typical Seyfert galaxies, but close to the present model $\left(M_{\mathrm{BH}}=2 \times 10^{6} M_{\odot}\right)$. In this section, we discuss the implications from a comparison between the multi-wavelength observations of the Circinus galaxy and the present model. All numerical results below are for the model with supernova feedback, if it is not explicitly stated otherwise.

\subsection{Dust Emission}

Using the snapshot of the radiation-hydrodynamic model shown in Section 3, we run 3D Monte Carlo radiative transfer simulations for dust emission, ${ }^{6}$ as performed in Sch14. Figure 5(a) shows SEDs for different viewing angles. As shown in Sch14, the SEDs are sensitive to the choice of the viewing angle. In the present case, the $10 \mu \mathrm{m}$ feature appears in the emission when the viewing angles $\theta_{v}$ are close to face-on $\left(\theta_{v}<30^{\circ}\right)$, but the feature appears in the absorption for $\theta_{v} \geqslant 60^{\circ}$. The model SED fits the Circinus galaxy (Prieto et al. 2010) fairly well if $\theta_{v} \gtrsim 70^{\circ}$ in a range between $\lambda \sim 2 \mu \mathrm{m}$ and $\lambda \sim 60 \mu \mathrm{m}$. Tristram et al. (2014) suggested that the nuclear disk-like dust emission revealed by VLT/MIDI has an inclination of $i \sim 75^{\circ}$, which is consistent with our SED analysis. $^{7}$

Figure 5(b) shows $12 \mu \mathrm{m}$ images observing the system from two directions, i.e., $75^{\circ}$ and edge-on. Notably, the cold molecular disk observed in Figure 1 is visible as a "clumpy dark lane" in these infrared images, which obscures the nuclear bright IR core. Without supernova feedback the scale height of the dense disk is not large enough to obscure the core. ${ }^{8}$

We also found that the dust emission forms a bright region that is elongated along the rotational axis with a dimmer counterpart in the $z<0$ direction. The structures are inhomogeneous and originate from the hot dust in the bipolar outflows. Recent MIR interferometric observations of nearby

\footnotetext{
6 The simulation code is RADMC-3D, http://www.ita.uni-heidelberg.de/ $\sim$ dullemond/software/radmc-3d/.

7 Ruiz et al. (2001) concluded that the radius of the dusty torus in the Circinus galaxy is $2-12 \mathrm{pc}$, which is consistent with our result.

8 The assumed supernova rate, i.e., $0.014 \mathrm{yr}^{-1}$ is about a factor of 10 larger than that suggested by the observed star formation rate in the Circinus galaxy (Hicks et al. 2009). However, the supernova feedback in numerical simulations with a sub-parsec resolution could be less efficient than in reality (Gentry et al. 2016).
} 
a
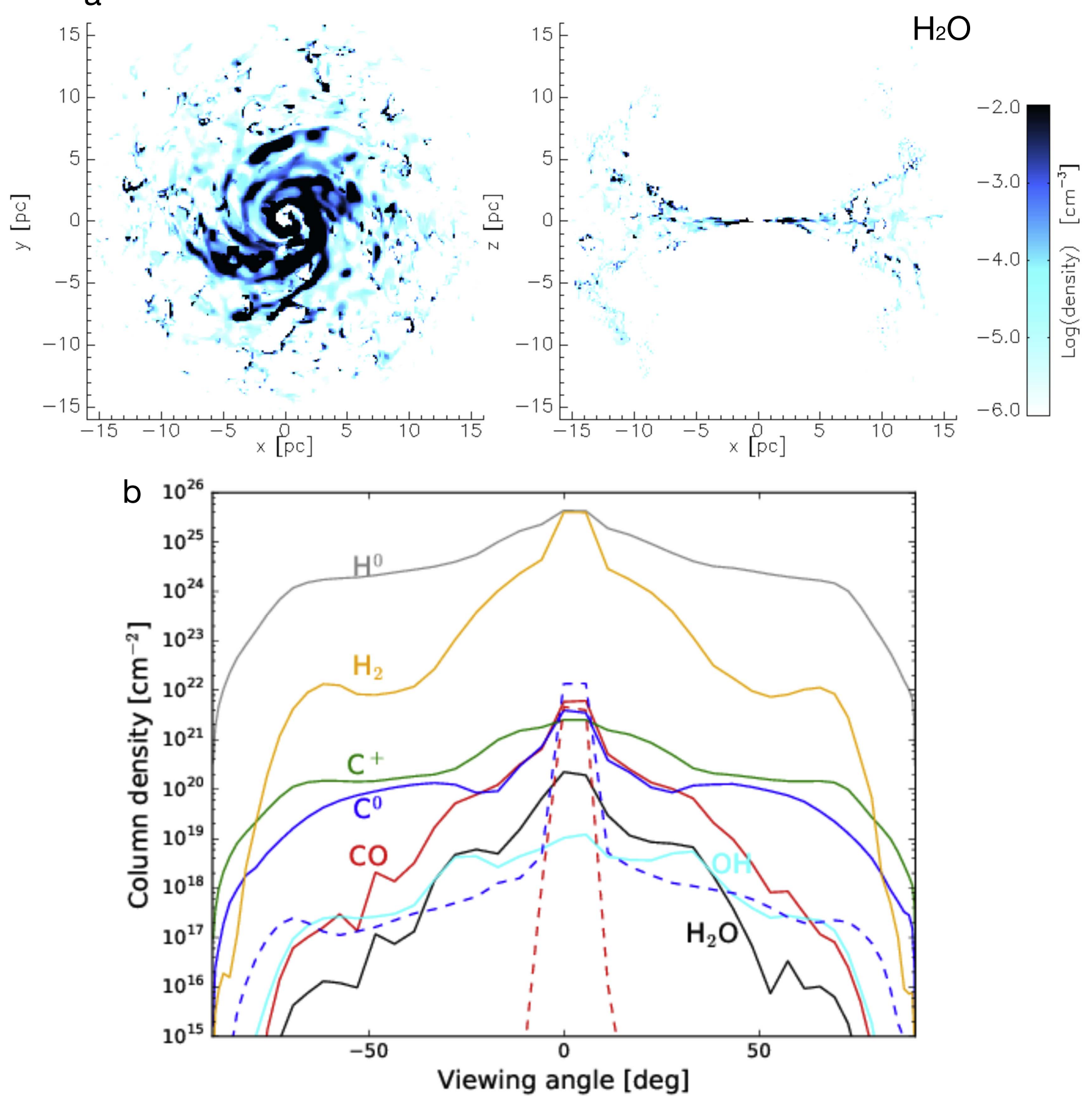

Figure 4. (a) Same as Figure 1, but for the number density distribution of $\mathrm{H}_{2} \mathrm{O}$. (b) Column density distribution as a function of the viewing angle $\left(0^{\circ}\right.$ means edge-on) for $\mathrm{H}^{0}, \mathrm{H}_{2}, \mathrm{CO}, \mathrm{C}^{0}, \mathrm{C}^{+}, \mathrm{OH}$, and $\mathrm{H}_{2} \mathrm{O}$. The dashed lines are the models without supernova feedback for $\mathrm{CO}$ (red) and $\mathrm{C}^{0}$ (blue).

Seyferts showed that the bulk of the MIR emission is emitted by dust in the polar region and not by the torus (Hönig et al. 2013; Tristram et al. 2014; Asmus et al. 2016; LópezGonzaga et al. 2016). Our results naturally expect the observed polar emission of dust. The hollow cones are not actually "empty." However, in the Circinus galaxy, Tristram et al. (2014) also detected a disk-like MIR emission. This might correspond to an inner part of the thin, dense disk seen in Figure 1 at $r<5 \mathrm{pc}$; however, additional physics and higher spatial resolution would be needed in order to study the 3D structures of this component in our simulations. Note that the size of the extended polar dust emission in Tristram et al.
(2014) is smaller (1-2 pc) than the polar emission in Figure 5(b). The MIDI observation could correspond to the innermost bright $12 \mu \mathrm{m}$ emission in our model. A more detailed comparison with the MIDI data will be discussed in a subsequent paper.

\subsection{Cold Molecular Gas}

Zhang et al. (2014) reported molecular gas in the nuclear region $(r<360 \mathrm{pc})$ with a kinetic temperature of $\sim 200 \mathrm{~K}$ and density of $\sim 10^{3.2} \mathrm{~cm}^{-3}$ using the multi- $J$ transition of ${ }^{12} \mathrm{CO}$ observed by the Atacama Pathfinder EXperiment (APEX) telescope. Figure 2 shows that there is a dominant phase of 


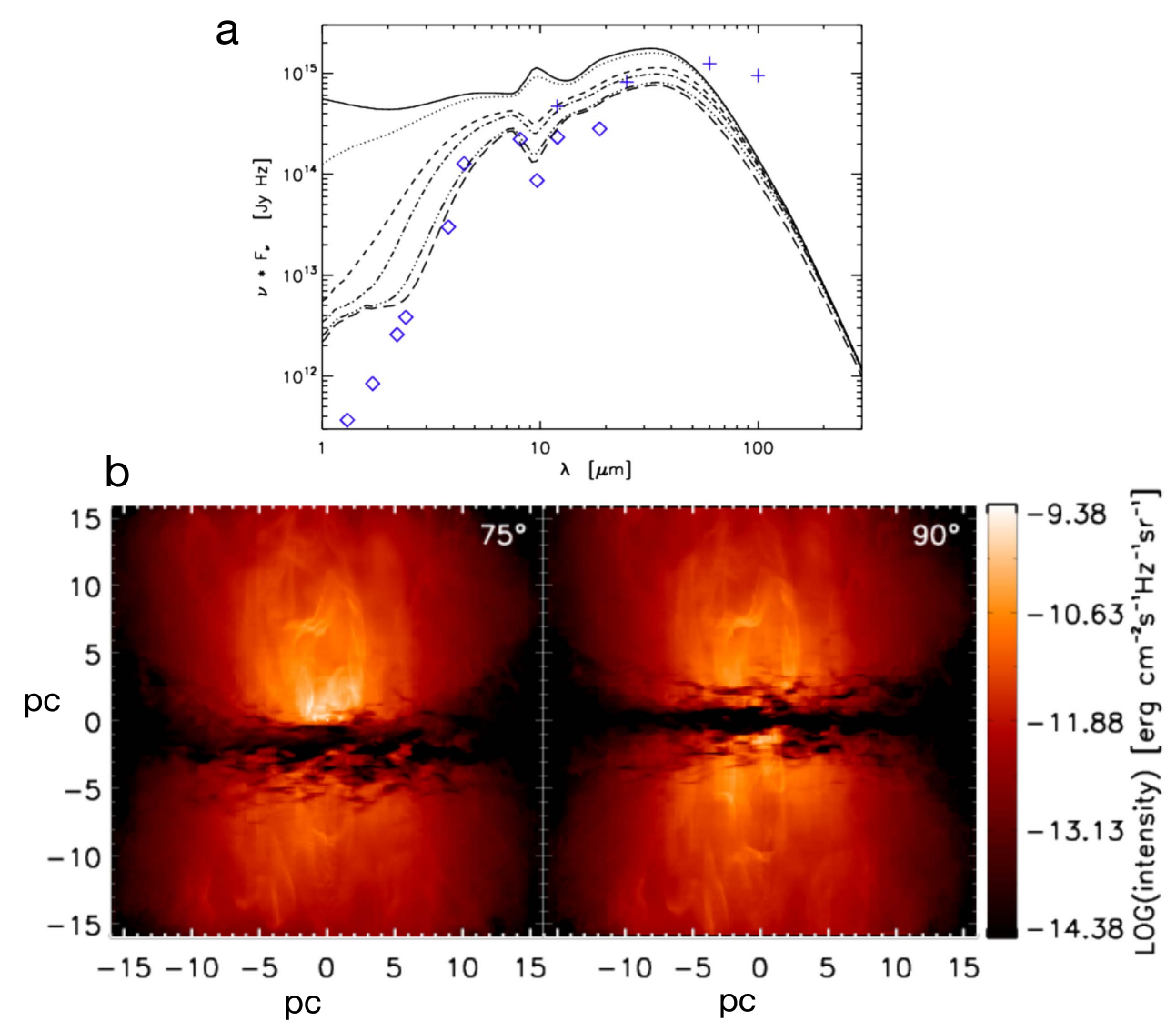

Figure 5. (a) Dust continuum radiative transfer SEDs for various inclination angles (top to bottom: $0^{\circ}, 30^{\circ}, 60^{\circ}, 70^{\circ}, 80^{\circ}, 90^{\circ}$ ) assuming a distance to the Circinus galaxy of $4.2 \mathrm{Mpc}$. The open diamonds refer to the observed nuclear SED, and the crosses represent large aperture IRAS data (compiled by Prieto et al. 2010). (b) Simulated $12 \mu \mathrm{m}$ images for an inclination angle of $75^{\circ}$ (left) and for the edge-on view (right). Labels are given in parsecs.

dense molecular gas, whose temperature correlates with density as $T_{\text {gas }} \sim 100 \mathrm{~K}\left(n / 10^{3} \mathrm{~cm}^{-3}\right)^{-1 / 2}$. For $T_{\text {gas }}=200 \mathrm{~K}$, gas density is $n \sim 2.5 \times 10^{2} \mathrm{~cm}^{-3}$. As shown in Figure 1(b), this high-density molecular gas forms a clumpy disk that is affected by energy feedback from the nuclear starburst. Origin of the warm molecular gas is not clear in the APEX result, but it can be verified using high-resolution observations with ALMA in the near future.

\subsection{Hot Molecular Gas}

The hot molecular gas $(1000-3000 \mathrm{~K})$ can be traced by the near-infrared region, such as $\mathrm{H}_{2}$ at $2.12 \mu \mathrm{m}$. Using VLT $/ \mathrm{NaCo}$, Mezcua et al. (2015) obtained $2.12 \mu \mathrm{m}$ images with a resolution of $0.09-0.16$ arcsec in the central $1 \mathrm{kpc}$ of seven nearby Seyfert galaxies. They found that the hot $\mathrm{H}_{2}$ is highly concentrated toward the central $100 \mathrm{pc}$ and that its morphology is often symmetrical. The mass fraction of hot $\mathrm{H}_{2}$ to the total cold gas mass in the central several tens of parsecs of nearby Seyfert galaxies is a few $\times 10^{-6}$. For example, in the Circinus galaxy, $M_{\text {hot }}=40 M_{\odot}$ for $r<26 \mathrm{pc}$. In our model, the total mass of hot $\mathrm{H}_{2}$ is $470 M_{\odot}$, and the total cold gas $(T \leqslant 40 \mathrm{~K})$ is $1 \times 10^{6} M_{\odot}$; therefore, the mass fraction is $4.7 \times 10^{-5}$ for $r<16 \mathrm{pc}$, a factor of 10 larger than the observation.

\subsection{Ionized Diffuse Gas and Compton-thick Material}

It is widely believed that the conical ionized gas structure (Prieto et al. 2005 and references therein) provides indirect evidence for the existence of a geometrically and optically thick tori. Our model, however, naturally reproduces a double hollow cone structure of ionized gas as a result of the radiationdriven outflows without a torus. The opening angle of the ionized gas is about $120^{\circ}$ (Figure 1(c)), which is similar to the observed ionization cone in the Circinus galaxy (Marconi et al. 1994; Veilleux \& Bland-Hawthorn 1997; Greenhill et al. 2003).

We found that the ionized gas in the biconical outflows is not uniform with $n \sim 10-100 \mathrm{~cm}^{-3}$ (Figure 1(c)). This is similar to the situation expected in a model of the coronal line region of Seyfert galaxies (e.g., Murayama \& Taniguchi 1998). The spectroscopic properties of the ionized gas in our models will be studied in detail by applying Cloudy (Ferland et al. 2013) to the hydrodynamic model in a subsequent paper.

Marinucci et al. (2013) found a Compton-thick, clumpy structure that is axisymmetric with respect to the nucleus of the Circinus galaxy, producing the cold reflection and the iron $\mathrm{K} \alpha$ line. They suggested that this could be the outer part of the dusty region responsible for the infrared emission. Arévalo et al. (2014) suggested that the "torus" has an equatorial column density $N_{\mathrm{H}}=6-10 \times 10^{24} \mathrm{~cm}^{-2}$, and the intrinsic $\mathrm{X}$-ray luminosity is $L_{2-10 \mathrm{keV}}=2.3-5.1 \times 10^{42} \mathrm{erg} \mathrm{s}^{-1}$ using NuSTAR, XMM-Newton, and Chandra. The column density and X-ray luminosity are consistent with our model. Our model shows that the Compton-thick gas $\left(N_{\mathrm{H}}>1.5 \times 10^{24} \mathrm{~cm}^{-2}\right)$ obscures the nucleus, if the viewing angle is $\sim 75^{\circ}$. Therefore, the cold reflection gas found in the X-ray observations would be the clumpy gas in the outer part of the circumnuclear disk shown in the $\mathrm{H}_{2}$ map (Figure 1(b)). 
The authors are grateful to the anonymous referee for constructive comments and suggestions. We thank Dr. Takuma Izumi for his valuable comments. Numerical computations were performed on a Cray XC30 at the Center for Computational Astrophysics at the National Astronomical Observatory of Japan. We thank C. P. Dullemond for making RADMC-3D publicly available and for his continuous support with the code. K.W. was supported by JSPS KAKENHI grant number $16 \mathrm{H} 03959$.

\section{REFERENCES}

Ádámkovics, M., Glassgold, A. E., \& Meijerink, R. 2011, ApJ, 736, 143 Aird, J., Coil, A. L., Georgakakis, A., et al. 2015, MNRAS, 451, 1892 Arévalo, P., Bauer, F. E., Puccetti, S., et al. 2014, ApJ, 791, 81

Asmus, D., Hönig, S. F., \& Gandhi, P. 2016, ApJ, 822, 109

Bernhard, E., Mullaney, J. R., Daddi, E., Ciesla, L., \& Schreiber, C. 2016, MNRAS, 460, 902

Buchner, J., Georgakakis, A., Nandra, K., et al. 2015, ApJ, 802, 89

Burlon, D., Ajello, M., Greiner, J., et al. 2011, ApJ, 728, 58

Chen, Y.-M., Wang, J.-M., Yan, C.-S., Hu, C., \& Zhang, S. 2009, ApJL, 695, L130

Davies, R. I., Müller Sánchez, F., Genzel, R., et al. 2007, ApJ, 671, 1388

Diamond-Stanic, A. M., \& Rieke, G. H. 2012, ApJ, 746, 168

Durré, M., \& Mould, J. 2014, ApJ, 784, 79

Esquej, P., Alonso-Herrero, A., González-Martín, O., et al. 2014, ApJ, 780, 86

Ferland, G. J., Porter, R. L., van Hoof, P. A. M., et al. 2013, RMxAA, 49, 137

Gentry, E. S., Krumholz, M. R., Dekel, A., \& Madau, P. 2016, MNRAS, submitted (arXiv:1606.01242)

Greenhill, L. J., Booth, R. S., Ellingsen, S. P., et al. 2003, ApJ, 590, 162

Hicks, E. K. S., Davies, R. I., Malkan, M. A., et al. 2009, ApJ, 696, 448

Hönig, S. F., Kishimoto, M., Tristram, K. R. W., et al. 2013, ApJ, 771, 87

Imanishi, M., Ichikawa, K., Takeuchi, T., et al. 2011, PASJ, 63, 447
Laor, A., \& Draine, B. T. 1993, ApJ, 402, 441

López-Gonzaga, N., Burtscher, L., Tristram, K. R. W., Meisenheimer, K., \& Schartmann, M. 2016, A\&A, 591, A47

Lusso, E., Hennawi, J. F., Comastri, A., et al. 2013, ApJ, 777, 86

Maloney, P. R., Hollenbach, D. J., \& Tielens, A. G. G. M. 1996, ApJ, 466, 561

Marconi, A., Moorwood, A. F. M., Origlia, L., \& Oliva, E. 1994, Msngr, 78, 20

Marconi, A., Risaliti, G., Gilli, R., et al. 2004, MNRAS, 351, 169

Marinucci, A., Miniutti, G., Bianchi, S., Matt, G., \& Risaliti, G. 2013, MNRAS, 436, 2500

Meijerink, R., \& Spaans, M. 2005, A\&A, 436, 397

Mezcua, M., Prieto, M. A., Fernández-Ontiveros, J. A., et al. 2015, MNRAS, 452,4128

Murayama, T., \& Taniguchi, Y. 1998, ApJL, 497, L9

Namekata, D., \& Umemura, M. 2016, MNRAS, 460, 980

Netzer, H. 1987, MNRAS, 225, 55

Prieto, M. A., Marco, O., \& Gallimore, J. 2005, MNRAS, 364, L28

Prieto, M. A., Reunanen, J., Tristram, K. R. W., et al. 2010, MNRAS, 402, 724

Ruiz, M., Efstathiou, A., Alexander, D. M., \& Hough, J. 2001, MNRAS, 325,995

Schartmann, M., Krause, M., \& Burkert, A. 2011, MNRAS, 415, 741

Schartmann, M., Wada, K., Prieto, M. A., Burkert, A., \& Tristram, K. R. W. 2014, MNRAS, 445, 3878 (Sch14)

Tristram, K. R. W., Burtscher, L., Jaffe, W., et al. 2014, A\&A, 563, A82

Ueda, Y., Akiyama, M., Hasinger, G., Miyaji, T., \& Watson, M. G. 2014, ApJ, 786, 104

Veilleux, S., \& Bland-Hawthorn, J. 1997, ApJL, 479, L105

Wada, K. 2001, ApJL, 559, L41

Wada, K. 2004, in Coevolution of Black Holes and Galaxies, ed. L. C. Ho (Cambridge: Cambridge Univ. Press), 187

Wada, K. 2012, ApJ, 758, 66 (W12)

Wada, K. 2015, ApJ, 812, 82 (W15)

Wada, K., \& Norman, C. A. 2002, ApJL, 566, L21 (WN02)

Wada, K., Papadopoulos, P. P., \& Spaans, M. 2009, ApJ, 702, 63

Woo, J.-H., Kim, J. H., Imanishi, M., \& Park, D. 2012, AJ, 143, 49

Xu, Y.-D. 2015, MNRAS, 449, 191

Zhang, Z.-Y., Henkel, C., Gao, Y., et al. 2014, A\&A, 568, A122 